\title{
Efficacy of mood stabilisers in the treatment of impulsive or repetitive aggression: systematic review and meta-analysis
}

Roland M. Jones, James Arlidge, Rebecca Gillham, Shuja Reagu, Marianne van den Bree and Pamela J. Taylor

\section{Background}

Individuals with repetitive or impulsive aggression in the absence of other disorders may be diagnosed with intermittent explosive disorder according to DSM-IV, but no such diagnostic category exists in ICD-10. Mood stabilisers are often used off-license for the treatment of aggression associated with a variety of psychiatric conditions, but their efficacy in these and in idiopathic aggression is not known.

\section{Aims}

To summarise and evaluate the evidence for the efficacy of mood stabilisers (anticonvulsants/lithium) in the treatment of impulsive or repetitive aggression in adults.

\section{Method}

A meta-analysis of randomised controlled trials that compared a mood stabiliser with placebo in adults without intellectual disability, organic brain disorder or psychotic illness, identified as exhibiting repetitive or impulsive aggression.

\section{Results}

Ten eligible trials (489 participants) were identified A pooled analysis showed an overall significant reduction in the frequency/severity of aggressive behaviour (standardised mean difference $(S M D)=-1.02,95 \% \mathrm{Cl}-1.54$ to -0.50 ), although heterogeneity was high $\left(l^{2}=84.7 \%\right)$. When analysed by drug type, significant effects were found in the pooled analysis of three phenytoin trials $(\mathrm{SMD}=-1.34,95 \% \mathrm{Cl}$ -2.16 to -0.52$)$, one lithium trial $(\mathrm{SMD}=-0.81,95 \% \mathrm{Cl}$ -1.35 to -0.28$)$, and two oxcarbazepine/carbamazepine trials $(\mathrm{SMD}=-1.20,95 \% \mathrm{Cl}-1.83$ to -0.56$)$. However, when the results of only those studies that had a low risk of bias were pooled (347 participants), there was no significant reduction in aggression $(\mathrm{SMD}=-0.28,95 \% \mathrm{Cl}-0.73$ to 0.17 , $\left.r^{2}=71.4 \%\right)$.

\section{Conclusions}

There is evidence that mood stabilisers as a group are significantly better than placebo in reducing aggressive behaviour, but not all mood stabilisers appear to share this effect. There is evidence of efficacy for carbamazepine/ oxcarbazepine, phenytoin and lithium. Many studies, however, were at risk of bias and so further randomised controlled trials are recommended.

\section{Declaration of interest}

R.M.J. has been paid for delivering educational presentations for Eli Lilly and Company.
Aggression is a normal human behaviour, but the ability to control aggressive impulses may be impaired or lost in a variety of mental disorders or after brain injury. People may also present in the absence of such disorders with repeated, discrete episodes of aggressive acts that do not appear to have been externally provoked (or are out of proportion to any such provocation) and are reportedly because of a failure to resist internally driven aggressive impulses. The DSM-IV ${ }^{1}$ attaches a specific diagnostic category of intermittent explosive disorder to such idiopathic aggression, although the ICD-10 $0^{2}$ does not. The DSM-IV approach, which built on the transformation of USA diagnostic behaviour with DSM-III, ${ }^{3}$ is still somewhat controversial, ${ }^{4}$ not least because it was seen as in part promotional of pharmaceutical interventions.

Anticonvulsants and lithium (as a group known as mood stabilisers) have long been reported to have utility in the treatment of aggression in individuals with epilepsy ${ }^{5}$ and those with intellectual disability, ${ }^{6,7}$ but clear evidence for their effectiveness is lacking. Despite the lack of evidence, recent reports suggest that mood stabilisers are frequently used off-license for this indication in hospital settings. A survey of psychiatric in-patients in the UK found that nearly a third were being prescribed at least one mood stabiliser, and in $41 \%$ of these cases, the main reason given by the prescriber was to control aggression. ${ }^{8}$

Previous systematic reviews of mood stabilisers are not optimally informative regarding the treatment of aggression. Several reviews have been conducted that focused on individuals with borderline personality disorder, and even in this fairly homogeneous group, the findings conflict. ${ }^{9,10}$ In these reviews, studies were included in which the treatment of aggression was not the primary aim, and therefore they included individuals who did not have high baseline levels of aggression. Another recent systematic review of anticonvulsant drugs, by definition excluding lithium, did not carry out a meta-analysis of their efficacy in reducing aggression due to concerns that the distributions of the data were skewed, and therefore conventional meta-analytic techniques may be inappropriate. ${ }^{11}$ However, appropriate statistical methods are available for transforming summary data, which can then be used reliably for further analysis.

The aims of this review were to conduct a systematic review and meta-analysis of the effectiveness of mood stabilisers for repeated or excessive aggression: using well-defined criteria for aggression, including but not confined to intermittent explosive disorder; based on randomised placebo-controlled trials of adults recruited specifically for the treatment of that aggression, with any mood stabiliser, including lithium.

\section{Method}

\section{Definition of aggression}

We adopted the definition used by Berkowitz, that aggression refers to goal-directed motor behaviour that has a deliberate 
intent to harm or injure another object or person. ${ }^{12}$ This definition includes verbal and physical acts of aggression, and excludes anger or hostility where there has been no physical act. We required that aggression was measured prospectively (sometimes categorised as 'state' aggression), rather than measured retrospectively or globally (or 'trait' aggression). We have included only studies in which participants were selected for the trial as having predefined problems with impulsive or repetitive aggression.

\section{Search strategy and selection}

We searched for studies, published in English, of randomised placebo-controlled trials of any duration that compared mood stabilisers with placebo for the treatment of impulsive aggression in adults using a comprehensive search strategy (see the online supplement to this paper). We excluded studies of individuals with intellectual disability and neurodegenerative disorders, and studies that did not recruit individuals specifically for the treatment of aggression. We did not exclude studies in which individuals had a personality disorder or substance use disorder, providing they were recruited for the primary purpose of treating repeated, impulsive aggression.

We searched MEDLINE, Embase, PsycINFO and the Cochrane Central Register of Controlled Trials (CENTRAL) from inception (accessed November 2009) using a comprehensive search strategy (online supplement). We also searched the System for Information of Grey Literature (SIGLE), ISI Web of Science conference proceedings, Index to Thesis/Electronic Thesis Online Service and Google Scholar (1989-November 2009). In addition, we searched the citations of all references retrieved. Two authors (J.A. and R.G.) independently reviewed the titles and abstracts obtained from the electronic searches. The full articles were obtained for further scrutiny where indicated. Disagreements were resolved by discussion with a third author (R.M.J.). For the majority of included studies we were able to extract sufficient information from the published findings; for the remainder, we contacted the authors and requested raw data.

\section{Outcome measures}

We used the frequency and/or severity of aggressive behaviour as the primary outcome measure, the latter being a total or global score derived from both frequency and severity of aggressive behaviour.

\section{Data extraction and assessment of risk of bias}

Two authors independently extracted data using a predesigned and piloted template. The same two authors independently assessed risk of bias for each study with respect to randomisation, masking and description of withdrawals, scored according to the Jadad scale, ${ }^{13}$ as well as for risk of bias because of selective reporting of results or failure to account adequately for individuals dropping out of the study (specifically whether an intention-to-treat analysis was carried out). Studies were categorised as at 'high' risk of bias if they had a Jadad score of two or less, or if there was evidence of selective reporting of results, or no intention-to-treat analysis. Otherwise, they were categorised as 'low' risk of bias.

\section{Data synthesis and analysis}

We calculated standardised mean differences (SMDs) in aggression scores between the intervention and placebo groups at the end of the trial. We analysed cross-over studies as having a parallel design. We carried out a priori subgroup analysis of studies by drug group (phenytoin, carbamazepine/oxcarbazepine, valproate/divalproex, levetiracetam and lithium). For studies that reported outcomes at more than one time point, we selected the final time point for analysis.

Distributions for which the standard deviation divided by the mean was $<1$ were considered skewed. ${ }^{14}$ Visual inspection of the distributions of individual study data made available to us (four studies) showed distributions that were skewed to the left (i.e. approximating a log-normal distribution). As standard meta-analytic methods assume normality we calculated log transformation of the summary data for each study using appropriate methods ${ }^{15}$ and used these data for further analysis. We repeated all analysis using the untransformed summary data for comparison.

We calculated the proportion of statistical heterogeneity for each outcome (i.e. frequency and severity of aggression) using $I^{2}$. We undertook a random effect meta-analysis where substantial heterogeneity was found $\left(I^{2}>50 \%\right)$, and a fixed-effect meta-analysis elsewhere. We investigated factors that potentially contributed to heterogeneity using sensitivity analyses. In these sensitivity analyses, we first excluded each study in turn. We then investigated the effect of excluding studies that we considered had a high risk of bias, and studies that measured only the frequency and not severity of aggression. We used STATA (version 10 for Mac), including the METAN module for all analyses.

\section{Results}

\section{Study characteristics}

We identified 1726 potentially relevant records, of which only 52 referred to trials of mood stabilisers to treat aggression (Fig. 1). Of these, 29 were excluded as either they were not randomised controlled trials (RCTs, 18 studies) or because the patient group did not meet our inclusion criteria (5 studies were with adults with an intellectual disability and 6 with participants who had an organic illness or neurological injury). A further 13 studies were then excluded (online Table DS1) because the participants were not recruited primarily for the treatment of aggression (9 studies), there was no placebo control (1 study) or there was no measure of aggressive behaviour (3 studies). Categories for exclusion were mutually exclusive, but in practice, most of the excluded studies met more than one exclusion criterion. Ten studies therefore remained for detailed analysis (Table 1; see also online Table DS2 for a more detailed version).

Of the included studies, one study ${ }^{20}$ reported results of an extension of a previous one, ${ }^{19}$ and here we obtained individual patient data from the authors and calculated relevant summary outcome values on the basis of intention to treat. We obtained individual patient data from another study, ${ }^{21}$ and additional relevant summary values for two further studies. ${ }^{24,25}$ One trial ${ }^{21}$ had three treatment arms, which we have reported as separate studies in our results.

We were able to extract sufficient data from eight papers (a total of ten trials) to be used in the meta-analysis, which included 489 participants, 247 of whom received the active treatment. Four of the studies used phenytoin (all using a dose of $300 \mathrm{mg}$ per day), and one study each used lithium (serum levels $0.6-1 \mathrm{mEq} / \mathrm{l}$ ), divalproex (serum levels maintained between $80-120 \mu \mathrm{g} / \mathrm{ml}$ ); valproate (dose $750 \mathrm{mg} /$ day), carbamazepine (dose $450 \mathrm{mg} /$ day); oxcarbazepine (dose 1200-2400 mg/day) and levetiracetam (dose $1000-3000 \mathrm{mg} /$ day). All but two studies ${ }^{16,17}$ had a Jadad score of three or above.

Only three studies ${ }^{23-25}$ clearly reported results using intentionto-treat analysis, and we were able to calculate values using intention-to-treat analysis from one further study reported across 







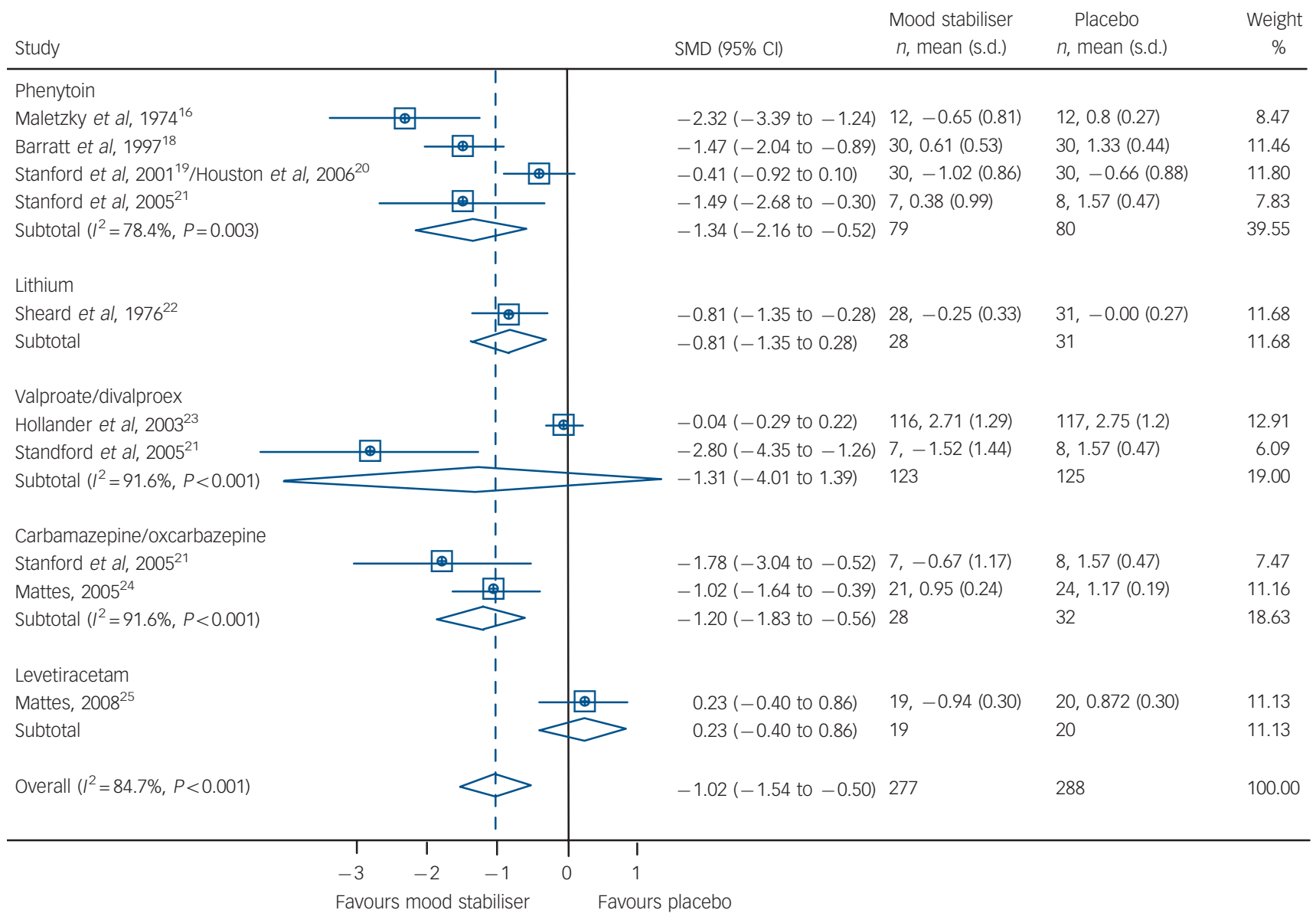

Fig. 2 Forrest plot of studies of randomised placebo-controlled studies of mood stabilisers for the treatment of aggression.

SMD, standardised mean difference.

untransformed data and found that the reduction in the severity of aggressive behaviour remained significant (overall $\mathrm{SMD}=-0.82,95 \% \mathrm{CI}-1.29$ to $\left.-0.36, I^{2}=81.7 \%\right)$. When analysed by drug type, significant effects were found in the pooled analysis of the three phenytoin trials $(\mathrm{SMD}=-1.34,95 \% \mathrm{CI}$ -2.16 to -0.52$)$, in the one lithium trial (SMD $=-0.81,95 \%$ CI -1.35 to -0.28$)$, and the two oxcarbazepine/carbamazepine trials $(\mathrm{SMD}=-1.20,95 \% \mathrm{CI}-1.83$ to -0.56$)$. No reduction in aggression was found for the pooled results of the two valproate studies or for the one levetiracetam study.

\section{Sensitivity analysis}

We removed each study in turn and found little change in heterogeneity in pooled analyses. We then analysed only studies that reported frequency of aggression as the outcome measure. The pooled results of these studies ${ }^{16,18-20,22}$ showed a significant effect of treatment over placebo on aggression $(\mathrm{SMD}=-1.1$, $95 \%$ CI -1.75 to $-0.46, I^{2}=76.6 \%$ ). Similarly, a significant relationship was also found between mood stabiliser medication and aggression in the six studies that reported global or total severity of aggression ${ }^{16,18,21,23-25}$ (pooled estimate; $\mathrm{SMD}=-1.20,95 \% \mathrm{CI}-1.90$ to $-0.49, I^{2}=87.8 \%$ ).

We then analysed only those studies in which we considered the risk of bias to be low (i.e. studies that we determined had a Jadad score of three or more, and had used intention-to-treat analysis). There was one study each that had investigated phenytoin, ${ }^{19,20}$ valproate, ${ }^{23}$ oxcarbazepine ${ }^{24}$ and levitaracetam. ${ }^{25}$
Of these, only the study into oxcarbazepine alone showed a significant reduction in aggression $(S M D=-1.02,95 \%$ CI -1.64 to -0.39 ). The pooled results of these four studies (that included a total of 347 participants) showed no significant reduction in aggression ( $\mathrm{SMD}=-0.28,95 \% \mathrm{CI}-0.73$ to 0.17 , $I^{2}=71.4 \%$; Fig. 3).

\section{Adverse events}

Two studies reported headache $e^{20,21,25}$ as a side-effect, causing discontinuation in one participant. One study reported high rates of sedation (65\%) and dizziness (20\%). ${ }^{25}$ Another study reported nausea, asthenia, increased appetite, depression, tremor and nervousness as side-effects. ${ }^{23}$ Side-effects were reported to be minor but were not detailed in two studies, ${ }^{24,18}$ and were not mentioned in three other studies.

\section{Discussion}

\section{Summary of evidence}

The current study reviewed randomised placebo-controlled trials that used a mood stabiliser to treat aggressive behaviour. We found evidence that treatment reduced either the frequency or severity of aggressive behaviour. There was evidence for significant reductions in aggression for those taking phenytoin, lithium and carbamazepine/oxcarbazepine, but not valproate or levetiracetam. Almost all of the studies reported adequate methods of randomisation and concealment, but many suffered from a risk 


\begin{tabular}{|c|c|c|c|c|c|c|}
\hline \multicolumn{3}{|l|}{ Study } & \multirow[t]{2}{*}{ SMD $(95 \% \mathrm{Cl})$} & \multirow[t]{2}{*}{$\begin{array}{l}\text { Mood stabiliser } \\
n \text {, mean (s.d.) }\end{array}$} & \multirow[t]{2}{*}{$\begin{array}{c}\text { Placebo } \\
n \text {, mean (s.d.) }\end{array}$} & \multirow[t]{2}{*}{$\begin{array}{l}\text { Weight } \\
\%\end{array}$} \\
\hline & 1 & & & & & \\
\hline Stanford et al, $2001^{19} /$ Houston et al, $2006^{20}$ & $\begin{array}{r}1 \\
1\end{array}$ & - & $-0.41(-0.92$ to 0.10$)$ & $30,-1.02(0.86)$ & $30,-0.66(0.88)$ & 24.78 \\
\hline Hollander et al, $2003^{23}$ & 1 & - & $-0.04(-0.29$ to 0.22$)$ & $116,2.71(1.29)$ & $117,2.75(1.2)$ & 32.54 \\
\hline Mattes, $2008^{25}$ & $\begin{array}{l}1 \\
1 \\
1 \\
1\end{array}$ & $\bullet$ & $0.23(-0.40$ to 0.86$)$ & $19,0.94(0.30)$ & $20,0.87(0.30)$ & 21.27 \\
\hline Mattes, $2005^{24}$ & 田 & & $-1.02(-1.64$ to -0.39$)$ & $21,0.95(0.24)$ & $24,1.17(0.19)$ & 21.41 \\
\hline Overall $\quad\left(I^{2}=71.4 \%, P<0.015\right)$ & $\begin{array}{l}1 \\
1 \\
1 \\
1 \\
1\end{array}$ & & $-0.28(-0.73$ to 0.17$)$ & 359 & 274 & 100.00 \\
\hline-2 & -1 & & 1 & & & \\
\hline
\end{tabular}

Fig. 3 Forrest plot of studies of randomised placebo-controlled studies of mood stabilisers for the treatment of aggression including only those studies with a low risk of bias.

SMD, standardised mean difference.

of bias as a result of inadequate reporting of individuals who had dropped out, particularly with lack of intention-to-treat analysis. When we included only those studies in which there was a low risk of bias, we found no pooled evidence of any significant benefit. When studies were analysed by drug category, only one category - oxcarbazepine - showed any significant effect, but this finding was based on only one study. Side-effects, where reported, tended to be mild; there were few cases of participants discontinuing the trial as a result of them.

Most of the studies were conducted with out-patients, and it is not clear if similar results would be found in other settings. The studies did not exclude individuals with a history of comorbid substance use disorders or personality disorders. This may have contributed towards heterogeneity, but has the benefit of being more representative of people with problem aggression.

\section{Limitations}

Heterogeneity between studies was high, and therefore it may be considered inappropriate to pool the results. We carried out subgroup analysis to mitigate the possible differential effect of different drug categories. With the exception of carbamazepine/ oxcarbazepine, drug categories showed high within-group heterogeneity (where there was more than one study in each category). We used random-effects meta-analysis, and performed sensitivity analyses that did not reveal any factors that contributed significantly towards heterogeneity. Our finding of a high level of heterogeneity is in itself useful as it emphasises that: evidence from studies to date is inconsistent; not all mood stabilisers are likely to have the same effect; and there may be important differences in patient characteristics or in the measurement of aggression between studies, which should be taken into account in future studies.

We included two studies in the analysis that had used a crossover design and analysed them as though they were derived from a parallel study, as data were not available to undertake a paired analysis. This is a conservative approach as it means that the cross-over studies would be relatively underweighted in the pooled analysis. Although methods are available for imputing values, we consider that our approach is robust and unlikely to significantly affect the results.

\section{Implications for practice}

Some mood stabilisers (oxcarbazepine, phenytoin and lithium) may well have a place in the treatment of aggressive behaviour for some individuals. The magnitude of the effect is difficult to determine, although any reduction in aggression may be considered a success in some patients. We used the standardised mean difference of log-transformed data as the principal summary measure. Although this is statistically appropriate, the interpretation of such a value in terms of the amount of change in aggression that would be expected in an individual is not straightforward. Moreover, group effects are likely to obscure considerable individual differences in response. This also has to be balanced against the potential side-effects of the medication, and the likelihood that treatment would need to be long term. We make no recommendation as to particular type of mood stabiliser, but to date, the best evidence of efficacy is available for carbamazepine/oxcarbazepine, phenytoin and possibly lithium. Given the limitations in the evidence currently available that prohibit conclusive findings of clear superiority of any of these mood stabilisers, we would recommend following general guidelines for psychopharmacological treatment, ${ }^{26}$ particularly to agree the length of time of trial of medication in advance, to titrate the dose, to objectively measure the response and to discontinue if there is no observed benefit.

\section{Recommendations for research}

These results highlight the need for further RCTs to investigate mood stabilisers for the treatment of aggression. We excluded all studies in which the primary aim was not the treatment of aggression, and as such, excluded several studies that reported 
good effect for other anti-epileptic or mood-stabilising drugs, especially in those with borderline personality disorder. These drugs include topiramate and lamotrigine, and we recommend that further studies are undertaken in other patient groups with these medications with the specific aim of targeting aggression. We also recommend that future studies use and report clear definitions of the inclusion criteria as well as the measure of aggression used. Several studies that we excluded measured anger as opposed to aggression. Although aggressive feelings and aggressive acts are likely to be correlated, it is the inability to control the aggressive feelings and to inhibit aggressive impulses that is impaired and this should be the focus of further study.

In conclusion, we found that there was an overall reduction in aggression in people treated with a mood stabiliser; however, many of the studies on which this review was based were at risk of bias, and therefore further RCTs are needed.

Roland M. Jones, MBChB, BSC, MSC, MRCPsych, Department of Psychological Medicine and Neurology, School of Medicine, Cardiff University, Cardiff and Llanarth Court Hospital, Abergavenny; James Arlidge, MB BS, BSc, Rebecca Gillham, Shuja Reagu, MB BS, MRCPSych, Marianne van den Bree, BSC, MSC, PhD, Pamela J. Taylor, MB BS, FRCPsych, MRCP, FMedSci, Department of Psychological Medicine and Neurology, School of Medicine, Cardiff University, Heath Park, Cardiff, UK

Correspondence: Roland M. Jones, Department of Psychological Medicine and Neurology, School of Medicine, Cardiff University, Heath Park, Cardiff CF14 4XN. Email: jonesrm6@cf.ac.uk

First received 25 May 2010, final revision 28 July 2010, accepted 11 Aug 2010

\section{Funding}

R.M.J. is supported by a Joint MRC/Welsh Assembly Clinical Research Training Fellowship (GO800450).

\section{References}

1 American Psychiatric Association. Diagnostic and Statistical Manual of Mental Disorders (4th edn) (DSM-IV). APA, 1994.

2 World Health Organization. The ICD-10 Classification of Mental and Behavioural Disorders: Clinical Descriptions and Diagnostic Guidelines. WHO, 1992.

3 American Psychiatric Association. Diagnostic and Statistical Manual of Mental Disorders (3rd edn) (DSM-IIII). APA, 1980.

4 Mayes R, Horwitz AV. DSM-III and the revolution in the classification of mental illness. J Hist Behav Sci 2005; 41: 249-67.

5 Ross AT, Jackson V. Dilantin sodium: its influence on conduct and on ratings of institutionalized epileptics. Ann Intern Med 1940; 14: 770-3.

6 Worrall EP, Moody JP, Naylor GJ. Lithium in non-manic-depressives: antiaggressive effect and red blood cell lithium values. Br J Psychiatry 1975; 136: 464-8.
7 Dale PG. Lithium therapy in aggressive mentally subnormal patients Br J Psychiatry 1980; 137: 469-74.

8 Haw C, Stubbs J. A survey of the off-label use of mood stabilizers in a large psychiatric hospital. J Psychopharmacol 2005; 19: 402-7.

9 Mercer D, Douglass AB, Links PS. Meta-analyses of mood stabilizers, antidepressants and antipsychotics in the treatment of borderline personality disorder: effectiveness for depression and anger symptoms. J Pers Disord 2009; 23: 156-74.

10 Nose M, Cipriani A, Biancosino B, Grassi L, Barbui C. Efficacy of pharmacotherapy against core traits of borderline personality disorder: meta-analysis of randomized controlled trials. Int Clin Psychopharmacol 2006; 21: 345-53.

11 Huband N, Ferriter M, Nathan R, Jones H. Antiepileptics for aggression and associated impulsivity. Cochrane Database Syst Rev 2010; 2: CD003499.

12 Berkowitz L. Aggression: It's Causes, Consequences, and Control. McGraw-Hill, 1993.

13 Jadad AR, Moore RA, Carroll D, Jenkinson C, Reynolds DJ, Gavaghan DJ, et al. Assessing the quality of reports of randomized clinical trials: is blinding necessary? Control Clin Trials 1996; 17: 1-12.

14 Altman DG, Bland JM. Detecting skewness from summary information. BMJ 1996 313: 1200.

15 Higgins JP, White IR, Anzures-Cabrera J. Meta-analysis of skewed data: combining results reported on log-transformed or raw scales. Stat Med 2008; 27: 6072-92.

16 Maletzky BM, Klotter J. Episodic dyscontrol: a controlled replication. Dis NerV Syst 1974; 35: 175-9.

17 Barratt ES, Stanford MS, Felthous AR, Kent TA. The effects of phenytoin on impulsive and premeditated aggression: a controlled study. J Clin Psychopharmacol 1991; 17: 341-9.

18 Barratt ES, Stanford MS, Kent TA, Felthous A. Neuropsychological and cognitive psychophysiological substrates of impulsive aggression. Biol Psychiatry 1997; 41: 1045-61.

19 Stanford MS, Houston RJ, Mathias CW, Greve KW, Villemarette-Pittman NR, Adams D. A double-blind placebo-controlled crossover study of phenytoin in individuals with impulsive aggression. Psychiatry Res 2001; 10: 193-203.

20 Houston RJ, Standford MS. Characterisation of aggressive behaviour and phenytoin response. Aggress Behav 2006; 32: 38-43.

21 Stanford MS, Helfritz LE, Conklin SM, Villemarette-Pittman NR, Greve KW, Adams D, et al. A comparison of anticonvulsants in the treatment of impulsive aggression. Exp Clin Psychopharmacol 2005; 13: 72-7.

22 Sheard $\mathrm{MH}$, Marini JL, Bridges $\mathrm{Cl}$, Wagner $\mathrm{E}$. The effect of lithium on impulsive aggressive behavior in man. Am J Psychiatry 1976; 133: 1409-13.

23 Hollander E, Tracy KA, Swann AC, Coccaro EF, McElroy SL, Wozniak P, et al. Divalproex in the treatment of impulsive aggression: efficacy in cluster B personality disorders. Neuropsychopharmacology 2003; 28: 1186-97.

24 Mattes JA. Oxcarbazepine in patients with impulsive aggression: a doubleblind, placebo-controlled trial. J Clin Psychopharmacol 2005; 25: 575-9.

25 Mattes JA. Levetiracetam in patients with impulsive aggression: a doubleblind, placebo-controlled trial. J Clin Psychiatry 2008; 69: 310-5.

26 Tyrer $\mathrm{P}$, Bateman AW. Drug treatment for personality disorders. Adv Psychiatr Treat 2004; 10: 389-98. 